\title{
Palpebral Infection with Bacillus Anthracis Complicated Cerebral Hemorrhagic Stroke: A Diagnosis not to be Ignored!
}

\section{Oujidi $\mathrm{Y}^{1 *}$, Bensaid $\mathrm{A}^{1}$, El melhaoui $\mathrm{I}^{2}$, Bkiyar $\mathrm{H}^{1}$ and Housni $\mathrm{B}^{1}$}

${ }^{1}$ Anaesthesia and Resuscitation Service, Hospital University Mohammed VI of Oujda, Faculty of Medicine and Pharmacy, Mohammed Premier University, Morocco

${ }^{2}$ Radiology department, Hospital University Mohammed VI of Oujda, Faculty of Medicine and Pharmacy, Mohammed Premier University, Morocco

*Corresponding author: Younes Oujidi, Anaesthesia and Resuscitation Service, Hospital University Mohammed VI of Oujda, Faculty of Medicine and Pharmacy, Mohammed Premier University, Morocco, Tel: +212762013707; Email: younesoujidi@gmail.com

\section{Abstract}

Anthrax is an anthropozoonosis due to a negative gram bacillus called Bacillus anthracis, palpebral localisation is rare in clinical practice and poses a problem of differential diagnosis, we report the case of a 5 year old girl who presented an under palpebral pustule evoluated into peri orbital ulceration with necrosis making suspect a palpebral localizations of anthrax the patient is complicated by CIVD associated with a hemorrhagic stroke [meningeal hemorrhage associated with a cerebral hematoma], the evolution was unfavorable with the death of the patient.

Keywords: Anthrax; Anthropozoonosis; CIVD; Hemorrhagic

\section{Introduction}

Palpebral charcoal is an anthropozoonosis little known to ophthalmologists, due to Bacillus anthracis. Human contamination is caused either by contact with sick animals or their contaminated products. Cutaneous anthrax is the most common clinical form, present in more than $95 \%$ of cases. Palpebral localization is rare in clinical practice and poses a differential diagnosis problem [1]. We report the case of a young patient who presented with a complicated palpebral anthrax of a hemorrhagic vascular accident and DIC.

\section{Case Report}

This is a 5-year-old patient with no significant medical history, from a rural area of Moroccan Oriental, who had a fever of $39,5^{\circ} \mathrm{C}$ associated with the development of a papula in the lower right eyelid and orbital burns.
The evolution was marked by the aggravation of the symptomatology by the installation of a palpebral right erythema motivating his consultation. At admission, the patient was conscious, hemodynamically and respiratory stable, febrile at $40^{\circ} \mathrm{C}$ with diffuse maculopapular exanthema with petechial purpuric elements. The ophthalmological examination was difficult given the impossibility of palpebral opening (Figure 1).

Biologically, shehad anemia at $9.4 \mathrm{~g} / \mathrm{dl}$, thrombocytopenia at 29,000 elements per $\mathrm{mm} 3$ and neutropenia at 1,180 29,000 elements per mm3. The prothrombin level was at $60 \%$, the activated cephalin time was at $1.2 \mathrm{~GB}$ and the PCR level was at 300. Renal function was normal. The cerebral and ophthalmic CT performed revealed multiple periorbital micro-collections without affecting the orbit or brain structures. Initial therapeutic management consisted of a triple antibiotic therapy combining ceftriaxone, 
metronidazole and gentamicin in resuscitation. The evolution was marked by the worsening of the periorbital lesion which became ulceronecrotic (Figure 2). The result of the blood culture revealed a bacillus anthracis susceptible only to Penicillin G. The diagnosis of anthrax was retained. Clinically, we know a rapid worsening of consciousness with a Glasgow score of $7 / 15$. The second cerebral CT scan performed revealed a spontaneous, non-traumatic intraparenchymal hematoma with surgical meningeal haemorrhage. Following neurovegetative signs of cerebral engagement, the patient died before surgery.

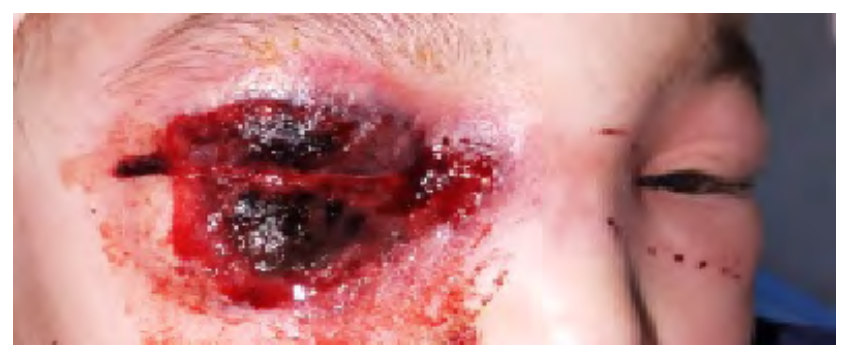

Figure 1: Maculopapular exanthema Periorbital.

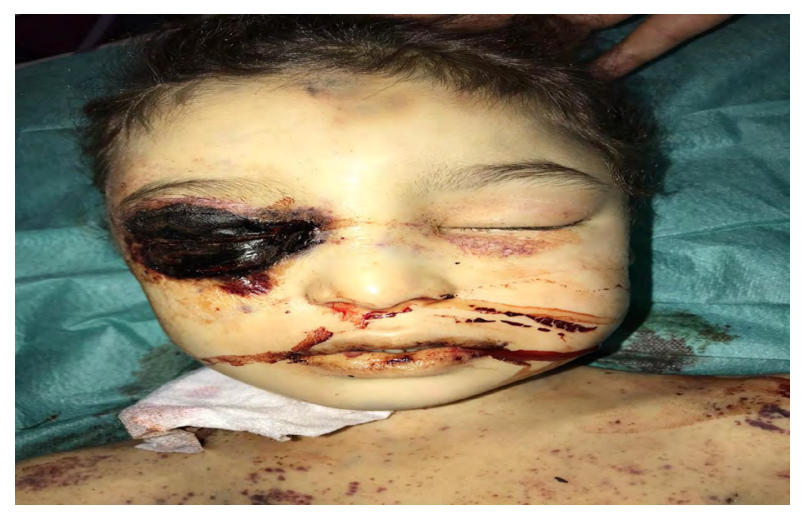

Figure 2: Periorbital lesion ulceronecrotic.

\section{Discussion}

Anthrax has been considered an important pathology throughout history and it is still relevant. This condition, little known to ophthalmologists, is characterized by its gravity and its rapid and deadly spread [2]. The pathogen is Bacillus anthracis [3]. The disease is caused by contact with diseased animals or their contaminated products. Bacillus anthracis spores are formed in the presence of oxygen or during exposure to air. They are extremely resistant, able to remain in animal products or in the soil for decades [2]. Some countries are considered endemic by the WHO, namely Asia, Africa, some European and American countries and parts of Australia [4]. In Morocco, this anthropozoonosis occurs in the form of small outbreaks; 72 cases were recorded between 2003 and 2007 [5].Transmission can take place in several modes, mostly transcutaneous, which explains the predominance of skin forms, which account for $95 \%$ of the forms [6]. Other modes of transmission are represented by the respiratory and digestive tract, some authors have raised the possibility of transmission by insect bite [7]. Progression to sepsis or meningitis may occur especially in the absence of treatment [6]. Palpebral localization remains rare, [7] and can occur in three clinical forms: malignant pustule, malignant edema and bullous form.The pathophysiology of anthrax is complex because it is related to its antiphagocytic and toxicologic capacity. Bacillus anthracis secretes three proteins that combine in a certain way to produce two toxins: (1) the lethal toxin resulting from the combination of the protective antigen (AP) , the lethal factor (FL) which results in the death of the patient, and (2) the edematous toxin that produces edema, a toxin formed by the protective antigen and the edematous factor (FET). Lethal toxin is the virulence factor responsible for most aspects of systemic infection. Both toxins act through the protective antigen [8]. Malignant pustule: represents the most common form and is manifested, after an incubation of 2 to 10 days, by a painless erythematous and pruriginous papula of variable diameter ranging from 2 to $5 \mathrm{~mm}$ in diameter. A ring of small vesicles appears around the papule, which will increase in size and excoriate during the next 2 days, resulting in the formation of a regular ulcer with an edge 1 to $3 \mathrm{~cm}$ in diameter. The evolution will take place after a few days towards the necrosis and drying of the ulcer resulting in the formation of a characteristic black bedsore firmly attached to the underlying tissues and often surrounded by edema. The bedsore falls after 1 to 2 weeks, leaving a retracting scar that poses particular problems to the palpebral localization, especially static disorders and palpebral retraction with lagophthalmos and risk of corneal exposure [9]. Treatment of anthrax is based on the administration of ciprofloxacin and penicillin G. In the case of resistance to penicillin, chloramphenicol or griseofulvin may be administered. Somes cases of resistance to third-generation cephalosporins have also been reported. Corticosteroid therapy is indicated for malignant edema. Surgical treatment of palpebral complications is performed by integumentary grafting. Prophylactic vaccination is recommended [10]. Concerning our patient, the treatment initially consisted of a triple antibiotic therapy with an unfavourable evolution marked by the installation of complications such as DIC and meningeal haemorrhage with a cerebral hematoma preceding the death of the patient.

\section{Conclusion}

The palpebral localization of anthrax disease is rare and should be suspected in any patient with black bedsore with perilesional edema especially in an evocative context (epidemic region at risk). The diagnosis confirmation 


\section{Clinical Dermatology Open Access Journal}

is provided by the isolation of Bacillus anthracis to the bacteriological examination usually by a sample taken at the level of the ulcer. Curative treatment is based on appropriate antibiotic therapy [Peni G] often leading to complete healing. It is a notifiable disease and only preventive measures will limit the health and economic impact of this disease with a high potential for spread.

\section{References}

1. Munteanu M, Munteanu G, Giuri S (2002) Anthrax cutané palpébral : à propos d'une observation. J Fr Ophtalmol 25: 940-943.

2. Sayouti A, Benhaddou R, Khoumiri R (2007) Charbon palpébral : à propos d'un cas. J Fr Ophtalmol 30: 403463.

3. Vaissaire J, Mock M, Doujet C (2001) Le charbon bactéridien : Epidémiologie de la maladie en France. Méd Mal Infect 31: 257-717.

4. Turnbull PCB (1998) Guidelines for the surveillance and control of anthrax in humans and animals. Geneva,
Switzerland. World Health Organization, Department of Communicable.

5. Ministère de la Santé, Rabat, Maroc. Bulletin épidémiologique, Archives du service des maladies épidémiques, 2003-2007.

6. Swartz MN (2001) Recognition and management of anthrax - an update. N Engl J Med 345(22): 1621-1626.

7. Celebi S, Aykan U, Alagoz G, Esmerligil S (2001) Palpebral anthrax. Eur J Ophthalmol 11(2): 171-174.

8. Mock M, Fouet A (2001) Toxines et pathogénie bactérienne. Annu Rev Microbiol 55: 647-671.

9. Hafidi Z, Handor H, Laghmari M, Handor N, Cherkaoui LO, et al. (2013) Diagnostic et traitement de la Maladie du charbon à localisation palpébrale : à propos d'un cas et revue de littérature. Pan African Medical Journal 15: 94.

10. Saysal HG, Kiratli H, Recep OF (2001) Anthrax as the cause of preseptal cellulitis and cicatricial ectropion. Acta Ophthalmol Scand 79: 208-209. 